\title{
The Squaring Operations in the Eilenberg-Moore Spectral Sequence and the Classifying Space of an Associative $H$-Space, I
}

\author{
By \\ Mamoru MimurA* and Masamitsu MORI**
}

\section{§. Introduction}

Let $G$ be a compact, connected, simple Lie group. Let $p$ be a prime. Consider $\{G ; p\}$ the set of all compact, associative $H$-spaces $X$ such that $H^{*}\left(X ; Z_{p}\right) \cong H^{*}\left(G ; Z_{p}\right)$ as Hopf algebras over the Steenrod algebra $\mathcal{A}_{p}$. (Remark that we do not require the existence of any map between $X$ and $G$ inducing the isomorphism.) As is well known, $X$ has the the classifying space $B X$ (see for example [8]).

The Eilenberg-Moore spectral sequence for $X$

$$
\begin{gathered}
E_{2}(X)=\operatorname{Cotor}_{A}\left(Z_{p}, Z_{p}\right) \Rightarrow H^{*}\left(B X ; Z_{p}\right), \\
\text { where } A=H^{*}\left(X ; Z_{p}\right),
\end{gathered}
$$

is a machinery to calculate $H^{*}\left(B X ; Z_{p}\right)$. When $H_{*}(G ; Z)$ has no $p$ torsion, it is quite easy to obtain $H^{*}\left(B X ; Z_{p}\right)$. In fact, $\operatorname{Cotor}_{A}\left(Z_{p}, Z_{p}\right)$ is a polynomial algebra and the Eilenberg-Moore spectral sequence collapses. But when $H_{*}(G ; Z)$ has $p$-torsion, it is, in general, difficult to obtain the structure of $H^{*}\left(B X ; Z_{p}\right)$.

Let $E_{j}$ be the compact, 1-connected, simple, exceptional Lie group of rank $j(j=6,7)$. Recently, Kono-Mimura [6] and Kono-MimuraShimada [7] have determined the module structure of $H^{*}\left(B E_{j} ; Z_{2}\right) \quad(j=6$, 7). Their method was to calculate algebraically $\operatorname{Cotor}_{A}\left(Z_{2}, Z_{2}\right)$ and then to show the collapsing of the spectral sequence $(0,1)$ for $E_{j}$ by making use of the properties of $E_{j}$ as Lie groups.

The aim of this paper is to give a proof of the collapsing of the

Communicated by N. Shimada, December 20, 1976.

* Mathematical Institute, Yoshida College, Kyoto University, Kyoto 606, Japan.

** College of Education, University of the Ryukyus, Naha 903, Japan. 
spectral sequence (0.1) independently of the properties as Lie groups, namely, to show the collapsing of the spectral sequence (0.1) for $X_{j}$ of $\left\{E_{j} ; 2\right\} \quad(j=6,7)$. Our method is to make use of the relationship between the defferentials and the two kinds of the squaring operations in the spectral sequence, which was obtained by W. Singer [12].

We denote by $E_{0} H^{*}\left(B X ; Z_{2}\right)$ the bigraded, associated algebra of $H^{*}\left(B X ; Z_{2}\right)$ with respect to the filtration $F^{\rho} H^{*}\left(B X ; Z_{2}\right)$ in the sense of Eilenberg-Moore, that is,

$$
E_{0}^{p, q} H^{*}\left(B X ; Z_{2}\right)=F^{p} H^{\rho+q}\left(B X ; Z_{2}\right) / F^{p+1} H^{p+q}\left(B X ; Z_{2}\right) .
$$

We shall use the convention to identify the elements in $E_{0} H^{*}\left(B X ; Z_{2}\right)$ with those in $H^{*}\left(B X ; Z_{2}\right)$, since $E_{0} H^{*}\left(B X ; Z_{2}\right) \cong H^{*}\left(B X ; Z_{2}\right)$ as modules.

Our results are stated as follows.

Theorem A. For any $X_{6} \in\left\{E_{6} ; 2\right\}$,

$$
E_{0} H^{*}\left(B X_{6} ; Z_{2}\right) \cong Z_{2}\left[y_{4}, y_{6}, y_{7}, y_{10}, y_{18}, y_{32}, y_{34}, y_{48}\right] / R,
$$

as an algebra, where $R$ is the ideal generated by (3.7).

Theorem B. (i) In $H^{*}\left(B X_{6} ; Z_{2}\right)$ the following relations hold mod decomposables.

$$
\begin{aligned}
& S q^{2} y_{4}=y_{6}, S q^{1} y_{6}=y_{7}, S q^{4} y_{6}=y_{10}, S q^{8} y_{10}=y_{18}, \\
& S q^{16} y_{18}=y_{34}, S q^{16} y_{32}=y_{48} . \\
& \text { (ii) } H^{*}\left(B X_{6} ; Z_{2}\right) \text { is generated by } y_{4} \text { and } y_{32} \text { over } A_{2} .
\end{aligned}
$$

Theorem C. For any $X_{7} \in\left\{E_{7} ; 2\right\}$,

$$
\begin{gathered}
E_{0} H^{*}\left(B X_{7} ; Z_{2}\right) \cong Z_{2}\left[y_{4}, y_{8}, y_{7}, y_{10}, y_{11}, y_{18}, y_{19}, y_{34},\right. \\
\left.y_{35}, y_{64}, y_{68}, y_{67}, y_{96}, y_{112}\right] / R,
\end{gathered}
$$

as an algebra, where $R$ is the ideal generated by (3.9) and (3.10).

Theorem D. (i) In $H^{*}\left(B X_{7} ; Z_{2}\right)$ the following relations hold mod decomposables. 
(4) $S q^{2} y_{4}=y_{6}, S q^{1} y_{6}=y_{7}, S q^{4} y_{6}=y_{10}, S q^{1} y_{10}=y_{11}$,

$$
\begin{array}{cl} 
& S q^{8} y_{10}=y_{18}, S q^{1} y_{18}=y_{19}, S q^{16} y_{18}=y_{34}, S q^{1} y_{34}=y_{35}, \\
& S q^{32} y_{34}=y_{66}, S q^{1} y_{66}=y_{67}, S q^{32} y_{64}=y_{96}, S q^{16} y_{96}=y_{112} . \\
\text { (ii) } \quad H^{*}\left(B X_{7} ; Z_{2}\right) \text { is generated by } y_{4} \text { and } y_{64} \text { over } A_{2} .
\end{array}
$$

Needless to say, Theorems A, B, C, D give the module structure of $H^{*}\left(B E_{j} ; Z_{2}\right) \quad(j=6,7)$ over $\mathscr{A}_{2}$. These are simpler proof than those of [6] and [7].

Remark. Let $G_{2}$ and $F_{4}$ be the compact, 1-connected, simple exceptional Lie groups of rank 2 and 4 respectively. Let $X_{2} \in\left\{G_{2} ; 2\right\}$ and $X_{4} \in\left\{F_{4} ; 2\right\}$. The structure of $H^{*}\left(B X_{i} ; Z_{2}\right) \quad(i=2,4)$ over $\mathcal{A}_{2}$ is obtained more easily by our argument. We leave them to the reader.

The paper is organized as follows. In $\S 1$ we recollect the Singer's results on the two kinds of squaring operations in the Eilenberg-Moore spectral sequence. In $\S 2$ we review that these operations coincide with those defined algebraically on $\operatorname{Cotor}_{A}\left(Z_{2}, Z_{2}\right)$ through the isomorphism $E_{2} \cong \operatorname{Cotor}_{A}\left(Z_{2}, Z_{2}\right)$. In $\S 3$ we calculate squaring operations on $\operatorname{Cotor}_{A}\left(Z_{2}\right.$, $\left.Z_{2}\right)$ for $A=H^{*}\left(X_{6} ; Z_{2}\right)$ and $H^{*}\left(X_{7} ; Z_{2}\right) . \S 4$ and $\S 5$ show that the Eilenberg-Moore spectral sequences for $X_{6}$ and $X_{7}$ collapse and this leads us to our results. The final section, $\S 6$, will be used to prove a lemma which is used in $\S 5$.

\section{§ 1. Squaring Operations in the Eilenberg-Moore Spectral Sequence}

Let $S_{*}(T)$ denote the normalized singular $Z_{2}$-chain complex of a space $T$ with all vertices at the base point. Put $S^{*}(T)=\operatorname{Hom}\left(S_{*}(T)\right.$, $\left.Z_{2}\right)$.

Let $X$ be a connected, associative $H$-space and $B X$ the classifying space of $X[8]$. A special case of the dual statement to Théorème 3.1 of Moore [9] states that there is an isomorphism

$$
H^{*}\left(B X ; Z_{2}\right) \cong \operatorname{Cotor}_{S^{*}(X)}\left(Z_{2}, Z_{2}\right) \quad\left(\text { or } \operatorname{Ext}_{S_{*}(X)}\left(Z_{2}, Z_{2}\right)\right) .
$$

Let $K$ denote the coalgebra $S^{*}(X)$. Let $\bar{C}(K)$ denote the cobar con- 
struction of $K$, in which $\bar{C}^{s}(K)=\bar{K} \otimes \cdots \otimes \bar{K}$ (s-times) with $\bar{K}=\sum_{t>0} K^{t}$. Then $\bar{C}(K)$ is a double complex with the external differential induced from the coalgebra structure of $K$ and the internal differential induced from the differential in $K$. Let Tot $\bar{C}(K)$ denote the total complex of $\bar{C}(K)$. Then $\operatorname{Cotor}_{K}\left(Z_{2}, Z_{2}\right)$ is, by definition, the cohomology of Tot $\bar{C}(K)$. The total complex Tot $\bar{C}(K)$ has a filtration such that

$$
F^{r} \operatorname{Tot}^{n} \bar{C}(K)=\sum_{\substack{p+q=n \\ p \geq r}} \bar{C}^{p, q}(K),
$$

where the first index $p$ is the external degree and the second one $q$ is the internal degree. This gives rise to a spectral sequence $\left\{E_{r}\right\}$ such that

$$
E_{2} \cong \operatorname{Cotor}_{H^{*}\left(X ; Z_{2}\right)}\left(Z_{2}, Z_{2}\right) \Rightarrow H^{*}\left(B X ; Z_{2}\right) .
$$

We call the spectral sequence (1.2) the Eilenberg-Moore spectral sequence for $X$.

Remark. This is dual to the spectral sequence

$$
E^{2} \cong \operatorname{Tor}^{H_{n}\left(X ; Z_{2}\right)}\left(Z_{2}, Z_{2}\right) \Rightarrow H_{*}\left(B X ; Z_{2}\right),
$$

which is constructed in [9].

Now we recollect the Singer's results [12] for our purpose. Singer shows that products and squaring operations are defined in $\operatorname{Cotor}_{S^{+}(X)}\left(Z_{2}\right.$, $\left.Z_{2}\right)$ as well as in $H^{*}\left(B X ; Z_{2}\right)$ and the isomorphism (1.1) preserves them (Proposition 1.1 of [12 I], Proposition 7.1 of [12 II]). This enables us to introduce products and squaring operations in the EilenbergMoore spectral sequence.

Proposition 1. 1 (Propositions 1.2, 1.3, 1.5 of [12 I]). In the Eilenberg-Moore spectral sequence $\left\{E_{r}\right\}$ for an associative $H$-space $X$ the following properties hold:

(1) Each $E_{r}(r \geq 2)$ is a differential algebra and products on $E_{2}$ determine those on $E_{r}(r \geq 2)$.

(2) There are squaring operations

$$
\begin{aligned}
& S q^{k}: E_{r}^{p, q} \rightarrow E_{r}^{p, q+k} \quad(0 \leq k \leq q), \\
& S q^{k}: E_{r}^{p, q} \rightarrow E_{r}^{p+k-q, 2 q} \quad(k \geq q),
\end{aligned}
$$


and the squaring operations on $E_{2}$ determine those on $E_{r}(r \geq 2)$.

(3) Let $\rho: F^{p} H^{p+q}\left(B X ; Z_{2}\right) \rightarrow E_{\infty}^{p, q}$ be the natural projection.

For $u \in F^{p} H^{p+q}\left(B X ; Z_{2}\right)$ and $v \in F^{r} H^{*}\left(B X ; Z_{p}\right)$, we have

i) $u v \in F^{p+r} H^{*}\left(B X ; Z_{2}\right)$ and $\rho(u v)=\rho(u) \rho(v)$,

ii) if $0 \leq k \leq q$, then $S q^{k} u \in F^{p} H^{*}\left(B X ; Z_{2}\right)$ and $\rho S q^{k} u=S q^{k} \rho u$,

iii) if $q \leq k$, then $S q^{k} u \in F^{p+k-q}\left(B X ; Z_{2}\right)$ and $\rho S q^{k} u=S q^{k} \rho u$.

The operation $S q^{k}: E_{r}^{q, p} \rightarrow E_{r}^{p, q+k}$ will be called a vertical squaring operation and $S q^{k}: E_{r}^{p, q} \rightarrow E_{r}{ }^{p+k-q, 2 q}$ a diagonal squaring operation.

Proposition 1.2 (Proposition 1. 4 of [12 I]). Let $u \in E_{r}^{p, q}(r \geq 2)$.

i) If $k \leq q-r+1$, then $d_{r} S q^{k} u=S q^{k} d_{r} u$ in $E_{r}$.

ii) If $q-r+1 \leq k \leq q$, then $S q^{k} u$ survives to $E_{t}^{p, q \perp k}$, where $t=2 r$ $+k-q-1, S q^{k} d_{r} u$ survives to $E_{t}^{p+t, 2 q-2 r+2}$ and $d_{t}\left[S q^{k} u\right]=\left[S q^{k} d_{r} u\right]$.

iii) If $q \leq k$, then $S q^{k} u$ survives to $E_{t}^{p+k-q, 2 q}$, where $t=2 r-1$, $S q^{k} d_{r} u$ survives to $E_{t}^{p+t+k-q, 2 q-2 r+2}$ and $d_{t}\left[S q^{k} u\right]=\left[S q^{k} d_{r} u\right]$.

Remark. We sometimes regard the vertical operation $S q^{k}: E_{r}^{p, q}$ $\rightarrow E_{r}^{p, q+k}$ is zero if $k>q$. In this sense the differentials commute with vertical operations, i.e., $d_{r} S q^{k} u=S q^{k} d_{r} u$ in $E_{r}$ for every $k \geq 0$ and $r \geq 2$.

\section{§ 2. Squaring Operations on the $\mathbb{E}_{2}$-Term}

Let $X$ be an associative $H$-space. Put $A=H^{*}\left(X ; Z_{2}\right)$.

Proposition 2. 1 (Theorem 2. 2 of [10]).

$$
E_{2} \cong \operatorname{Cotor}_{4}\left(Z_{2}, Z_{2}\right) \text { as algebras . }
$$

We recall the two kinds of squaring operations on $\operatorname{Cotor}_{A}\left(Z_{2}, Z_{2}\right)$.

Let $\bar{C}(A)$ be the cobar construction of $A$. Let $\alpha=\left[x_{1}|\cdots| x_{p}\right]$ $\in \bar{C}^{p, q}(A)$. Define an operation $S q_{\gamma^{r}}^{k}: \bar{C}^{p, q}(A) \rightarrow \bar{C}^{p, q+k}(A)$ by

$$
S q^{k}{ }_{V} \alpha=\sum\left[S q^{k_{1}} x_{1}|\cdots| S q^{k_{p}} x_{p}\right], k_{1}+\cdots+k_{p}=k .
$$

Then $S q^{k}{ }$ commutes with the coboundary in $\bar{C}(A)$, since $A$ is the coalgebra over the Steenrod algebra. Hence this induces

$$
S q^{k}{ }_{V}: \operatorname{Cotor}_{A}^{p, q} \rightarrow \operatorname{Cotor}_{A}^{p, q+k} .
$$


Let $\bar{B}(A)$ be the bar construction of $A$, i.e.,

$$
\bar{B}^{s}(A)=\bar{H}_{*}\left(X ; Z_{2}\right) \otimes \cdots \otimes \bar{H}_{*}\left(X ; Z_{2}\right) \quad(s \text {-times }) .
$$

There is a map with external degree $i \geq 0$,

$$
\Delta_{i}: \bar{B}(A) \rightarrow \bar{B}(A) \otimes \bar{B}(A),
$$

satisfying $d \Delta_{i}+\Delta_{i} d=\Delta_{i-1}+T \Delta_{i-1} \quad\left(\Delta_{-1}=0\right)$. The cup-i-product

$$
\bigcup_{i}: \bar{C}^{p}(A) \otimes \bar{C}^{q}(A) \rightarrow \bar{C}^{p+q-i}(A)
$$

is defined by

$$
\begin{gathered}
\left(\alpha \bigcup_{i} \beta\right)(c)=(\alpha \otimes \beta) \Delta_{i}(c) \text { for } \alpha \in \bar{C}^{p}(A), \beta \in \bar{C}^{q}(A), \\
c c \in \bar{B}^{p+q-i}(A)
\end{gathered}
$$

and satisfies

$$
\delta\left(\alpha \bigcup_{i} \beta\right)=\delta \alpha \bigcup_{i} \beta+\alpha \bigcup_{i} \delta \beta+\alpha \bigcup_{i-1} \beta+\beta \bigcup_{i-1}^{\cup} \alpha .
$$

Then an operation $S q_{D}^{k}: \bar{C}^{p, q}(A) \rightarrow \bar{C}^{p+k, 2 q}(A)$ is defined by

$$
S q^{k} \alpha=\alpha \bigcup_{p-k} \alpha+\delta \alpha \bigcup_{p-k+1} \alpha \quad \text { for } \quad \alpha \in \bar{C}^{p, q}(A) .
$$

This commutes with the coboundary and induces

$$
S q_{D}^{k}: \operatorname{Cotor}_{A}^{p, q} \rightarrow \operatorname{Cotor}_{A}^{p+k, 2 q} .
$$

The construction of $S q^{k}{ }_{D}$ is essentially due to [1]. The explicit formula for the cup-i-product may be found in [14]. Especially, we recall the formulae:

$$
\begin{aligned}
& \quad\left[x_{1}|\cdots| x_{s}\right] \bigcup_{0}\left[x_{s+1}|\cdots| x_{s+r}\right]=\left[x_{1}|\cdots| x_{s+r}\right], \\
& {\left[x_{1}|\cdots| x_{s}\right] \underset{1}{\cup}\left[x_{s+1}|\cdots| x_{s+r}\right]=\sum_{i=1}^{s}\left[x_{1}|\cdots| x_{i-1}\left|x_{i}{ }^{(1)} x_{s+1}\right|\right.} \\
& \left.\quad \cdots\left|x_{i}{ }^{(r)} x_{s+r}\right| x_{i+1}|\cdots| x_{s}\right], \\
& {\left[x_{1}|\cdots| x_{s}\right] \bigcup_{s}\left[x_{1}|\cdots| x_{s}\right]=\left[x_{1}{ }^{2}|\cdots| x_{s}{ }^{2}\right],}
\end{aligned}
$$

where $\psi^{(r-1)}(x)=\sum x^{(1)} \otimes \cdots \otimes x^{(r)}, \psi^{(r-1)}: A \rightarrow A \otimes \cdots \otimes A$ ( $r$-times $)$, is the $(r-1)$-iterated diagonal map.

Proposition 2. 2 (Propositions 7.2, 7.3 of [12 II]). Through the 
isomorphism $E_{2} \cong \operatorname{Cotor}_{A}\left(Z_{2}, Z_{2}\right)$,

i) if $0 \leq k \leq q$, then the vertical squaring operation $S q^{k}$ on $E_{2}$ coincides with $S q^{k}{ }_{V}$ on $\operatorname{Cotor}_{A}\left(Z_{2}, Z_{2}\right)$,

ii) if $q \leq k$, then the diagonal squaring operation $S q^{k}$ on $E_{2}$ coincides with $S q^{k-q}{ }_{D}$ on $\operatorname{Cotor}_{1}\left(Z_{2}, Z_{2}\right)$.

Corollary 2.3. Let $\sum\left[x_{1}|\cdots| x_{p}\right] \in \bar{C}^{p, q}(A)$ and $\sum\left[x_{p+1}|\cdots| x_{p+r}\right]$ $\in \bar{C}^{r, s}(A)$ represent $u \in E_{2}^{p, q}$ and $v \in E_{2}^{r, s}$ respectively. Then

i) $\sum\left[x_{1}|\cdots| x_{p}\left|x_{p+1}\right| \cdots \mid x_{p+r}\right] \in \bar{C}^{p+r, q+s}(A)$ represents $u v \in E_{2}^{p+r, q+s}$,

ii) if $0 \leq k \leq q$, then

$$
\sum_{k_{1}, \cdots, k_{p}-k} \sum\left[S q^{k_{1}} x_{1}|\cdots| S q^{k_{p}} x_{p}\right] \in \bar{C}^{p, \eta+k}(A)
$$

represents $S q^{k} u=E_{2}{ }^{p, 4+h}$.

iii) if $q \leq k$, then

$$
\sum\left[x_{1}|\cdots| x_{p}\right] \underset{p-k \dashv p}{\cup}\left[x_{1}|\cdots| x_{p}\right] \in \bar{C}^{p+k-q, 2 q}
$$

represents $S q^{k} u \in{E_{2}}^{p+k-q, 2 q}$.

Proof. Immediate from Propositions 2.1, 2.2 and (2.1), (2.2).

q.e.d.

Here we remark, for later use:

Proposition 2.4. As for the vertical squaring operation, the Cartan formula holds on $E_{2}$, i.e.,

$$
S q^{k}(u v)=\sum_{i \vdash j=k} S q^{i} u S q^{j} v \text { for } u, v \in E_{2}
$$

and $E_{r}(r \geq 2)$ inherits this formula.

Proof. We confirm this by Corollary 2.3, i), ii), and Proposition 1. 1, (1), (2), though this may be proved by the standard argument.

q.e.d.

Let $\phi$ be the diagonal map of $A=H^{*}\left(X ; Z_{2}\right)$. Let $L$ be a quotient coalgebra of $\bar{A}$ over the Steenrod algebra $\mathcal{A}_{2}$ with projection $\theta: A \rightarrow L$. 
$\bar{\phi}^{\prime}$ denotes the diagonal map of $L$. Note that $L$ is not equipped with unit. Construct the tensor algebra $T(s L)$ with product $\psi$, where $s$ is the suspension, that is, the operation to make a copy with external degree added by one. Let $I$ be the two-sided ideal generated by $\psi \circ(s \theta \otimes s \theta)$ $\circ \phi(\operatorname{Ker} \theta)$. Let $\bar{X}=T(s L) / I$. The differential $\bar{d}$ on $\bar{X}$ is induced so that $\bar{d}=\psi \circ \bar{\phi}^{\prime} \circ s^{-1}: s L \rightarrow T(s L)$ is derivative. Then $\bar{d}(I) \subset I$ and $\bar{d} \circ \bar{d}=0$, and this is well-defined. $\bar{X}$ is the quotient of $\bar{C}(A)$ as differential algebra with projection $p: \bar{C}(A) \rightarrow \bar{X}$ such that $p\left[x_{1}|\cdots| x_{n}\right]=s \theta x_{1} \cdots s \theta x_{n}$ (see [11]). The (vertical) squaring operation on $\bar{X}$ is defined by

$$
S q^{k}{ }_{V} x=\sum_{k_{1}+\cdots+k_{n}=k} s S q^{k_{1}} x_{1} \cdots s S q^{k_{n}} x_{n}, x=s x_{1} \cdots s x_{n} \in T(s L)
$$

for $k \geq 0$.

Proposition 2. 5. The projection $p: \bar{C}(A) \rightarrow \bar{X}$ preserves the operation $S q_{v}^{k}$.

Proof. Immediate from Propositions 2. 1, 2.2 and Corollary 2. 3.

q.e.d.

Corollary 2. 6. Assume that $p: \bar{C}(A) \rightarrow \bar{X}$ induces an isomorphism on cohomology. Let $\sum s x_{1} \cdots s x_{p} \in \bar{X}^{p, q}$ represent $u \in E_{2}^{p, q}$. Then if $0 \leq k \leq q$, the element

$$
\sum_{k_{1}+\cdots+k_{p}=k} \sum s S q^{k_{1}} x_{1} \cdots s S q^{k_{p}} x_{p} \in \bar{X}^{p, q+k}
$$

represents $S q^{k} u \in E_{2}^{p, q+k}$.

Proof. Immediate from Corollary 2. 3 and Proposition 2. 5.

q.e.d.

\section{§ 3. Squaring Operations on $\operatorname{Cotor}_{A}$ for $A=H^{*}\left(X_{6} ; Z_{2}\right)$ and

$$
H^{*}\left(X_{7} ; Z_{2}\right)
$$

Let $X_{6} \in\left\{E_{8} ; 2\right\}$ and $X_{7} \in\left\{E_{7}, 2\right\}$.

By definition and [2], we have

$$
H^{*}\left(X_{6} ; Z_{2}\right)=Z_{3}\left[x_{3}\right] /\left(x_{3}{ }^{4}\right) \otimes \Lambda\left(x_{5}, x_{9}, x_{15}, x_{17}, x_{23}\right),
$$




$$
H^{*}\left(X_{7} ; Z_{2}\right)=Z_{2}\left[x_{3}, x_{5}, x_{9}\right] /\left(x_{3}{ }^{4}, x_{5}^{4}, x_{9}^{4}\right) \otimes \Lambda\left(x_{15}, x_{17}, x_{23}, x_{27}\right) .
$$

The reduced diagonal map is given by Theorem 3.1 of [6] and Theorem 1.8 of [7], namely,

$$
\begin{array}{ll}
\text { for } X_{6}, X_{7}, & \bar{\phi}\left(x_{i}\right)=0 \quad(i=3,5,9,17), \\
\text { for } X_{6}, & \bar{\phi}\left(x_{15}\right)=x_{9} \otimes x_{3}{ }^{2}, \\
& \bar{\phi}\left(x_{23}\right)=x_{17} \otimes x_{3}{ }^{2}, \\
\text { for } X_{7} . & \bar{\phi}\left(x_{15}\right)=x_{5} \otimes x_{5}{ }^{2}+x_{9} \otimes x_{3}{ }^{2}, \\
& \bar{\phi}\left(x_{23}\right)=x_{5} \otimes x_{9}{ }^{2}+x_{17} \otimes x_{3}{ }^{2}, \\
& \bar{\phi}\left(x_{27}\right)=x_{9} \otimes x_{9}{ }^{2}+x_{17} \otimes x_{5}{ }^{2} .
\end{array}
$$

The squaring operations on the elements are given by [2] and [13], namely,

(3. 4) for $X_{6}, X_{7}, \quad x_{5}=S q^{2} x_{3}, x_{9}=S q^{4} x_{5}, x_{17}=S q^{8} x_{9}, x_{23}=S q^{8} x_{15}$,

(3.5) for $X_{7}, \quad x_{27}=S q^{4} x_{23}$,

(3. 6) for $X_{6}, X_{7}, \quad x_{17}=S q^{2} x_{15}$.

Proposition 3.1. (i) Let $A=H^{*}\left(X_{6} ; Z_{2}\right)$. Then as an algebra, $E_{2} \cong \operatorname{Cotor}_{A}\left(Z_{2}, Z_{2}\right) \cong Z_{2}\left[y_{1}, y_{6}, y_{7}, y_{10}, y_{18}, y_{32}, y_{34}, y_{48}\right] / R$, where the gradings of generators are given by

$$
\begin{aligned}
& y_{4} \in(1,3), y_{6} \in(1,5), y_{7} \in(1,6), y_{10} \in(1,9), \\
& y_{18} \in(1,17), y_{32} \in(2,30), y_{31} \in(2,32), y_{48} \in(2,46),
\end{aligned}
$$

$\left(y \in(i, j)\right.$ means $\left.y \in E_{2}^{i, j}\right)$ and $R$ is the ideal generated by

$$
y_{7} y_{10}, y_{7} y_{18}, y_{7} y_{34}, y_{34}^{2}+y_{10}^{2} y_{48}+y_{18}^{2} y_{32} \text {. }
$$

(ii) The following relations hold in $E_{2}$ :

$S q^{2} y_{4}=y_{6}, S q^{1} y_{6}=y_{7}, S q^{4} y_{6}=y_{10}, S q^{8} y_{10}=y_{18}, S q^{16} y_{32}=y_{48}$.

Proof. The calculation of $\operatorname{Cotor}_{A}\left(Z_{2}, Z_{2}\right)$ is purely algebraic, and hence (i) follows from Theorem 2.3 of [6]. To determine the squaring operations, recall the outline of their calculation. Let $L=\left\{x_{3}, x_{5}, x_{3}{ }^{2}, x_{9}\right.$, $\left.x_{17}, x_{15}, x_{23}\right\}$ and denote the corresponding elements by $s L=\left\{a_{4}, a_{6}, a_{7}, a_{10}\right.$, 
$\left.a_{18}, b_{16}, b_{24}\right\}$. Then by (3. 4) we have

$$
S q^{2} a_{4}=a_{6}, S q^{1} a_{6}=a_{7}, S q^{4} a_{6}=a_{10}, S q^{8} a_{10}=a_{18}, S q^{8} b_{16}=b_{24} .
$$

Form a differential algebra $(\bar{X}, \bar{d})$ as in $\S 2$. Explicitly $\bar{X}$ is isomorphic to the polynomial algebra $Z_{2}\left[a_{4}, a_{6}, a_{7}, a_{10}, a_{18}, b_{16}, b_{24}\right]$. Then the projection $p: \bar{C}(A) \rightarrow \bar{X}$ induces an isomorphism on cohomology, i.e., $\operatorname{Cotor}_{A}\left(Z_{2}\right.$, $\left.Z_{2}\right) \cong H(\bar{X}, \bar{d})$. Each $y_{i}$ is represented in $\bar{X}$ as follows:

$$
\begin{aligned}
& y_{i}: a_{i}(i=4,6,7,10,18), \\
& y_{32}: b_{16}^{2}, \quad y_{48}: b_{24}^{2}, \quad y_{34}: a_{10} b_{24}+a_{18} b_{16} .
\end{aligned}
$$

Note that the squaring operations on $y_{i}$ follow immediate from Corollary 2. 6 and (3.8).

q.e.d.

We next turn to $X_{7}$.

Proposition 3. 2. (i) Let $A=H^{*}\left(X_{7} ; Z_{2}\right)$. Then as an algebra,

$$
\begin{gathered}
E_{2} \cong \operatorname{Cotor}_{d}\left(Z_{2}, Z_{2}\right) \cong Z_{2}\left[y_{4}, y_{6}, y_{7}, y_{10}, y_{11}, y_{18}, y_{19}, y_{34}, y_{35},\right. \\
\left.y_{66}, y_{67}, y_{64}, y_{96}, y_{112}\right] / R,
\end{gathered}
$$

where the gradings of generators are given by

$$
\begin{aligned}
& y_{4} \in(1,3), y_{6} \in(1,5), y_{7} \in(1,6), y_{10} \in(1,9), \\
& y_{11} \in(1,10), y_{18} \in(1,17), y_{19} \in(1,18), y_{34} \in(2,32), \\
& y_{35} \in(2,33), y_{66} \in(3,63), y_{67} \in(3,64), y_{64} \in(4,60), \\
& y_{96} \in(4,92), y_{112} \in(4,108),
\end{aligned}
$$

and $R$ is the ideal generated by

$$
\begin{aligned}
& y_{6} y_{11}+y_{10} y_{7}, y_{6} y_{19}+y_{18} y_{7}, y_{10} y_{19}+y_{18} y_{11}, \\
& y_{11}^{3}+y_{19} y_{7}^{2}, y_{11} y_{19}^{2}, y_{19}^{3}, y_{7} y_{34}+y_{6} y_{35}+y_{19} y_{11}^{2}, \\
& y_{11} y_{34}+y_{10} y_{35}+y_{7} y_{19}^{2}, y_{19} y_{34}+y_{18} y_{35}, y_{11} y_{35}^{2}+y_{7}^{2} y_{67}, \\
& y_{19} y_{35}^{2}+y_{11}^{2} y_{67}, y_{19}^{2} y_{67}, y_{34}^{4}+y_{18}^{4} y_{64}+y_{10}^{4} y_{96}+y_{6}^{4} y_{112}, \\
& y_{35}^{4}+y_{19}^{4} y_{64}+y_{11}^{4} y_{96}+y_{7}^{4} y_{112}, y_{66}^{2}+y_{10}^{2} y_{112}+y_{18}^{2} y_{96}, \\
& y_{67}^{2}+y_{11}^{2} y_{112}+y_{19}^{2} y_{96},
\end{aligned}
$$


(3. 10)

$$
\begin{aligned}
& y_{7} y_{66}+y_{6} y_{67}+y_{19}^{2} y_{35}, \quad y_{11} y_{66}+y_{10} y_{67}, \quad y_{19} y_{66}+y_{18} y_{67}, \\
& y_{35}^{2} y_{67}+y_{7}^{2} y_{11} y_{112}+y_{11}^{2} y_{19} y_{96}, \\
& y_{34} y_{67}+y_{35} y_{66}, \quad y_{66} y_{67}+y_{10} y_{11} y_{112}+y_{18} y_{19} y_{96}, \\
& y_{34}^{2} y_{35}^{2}+y_{18}^{2} y_{19}^{2} y_{64}+y_{10}^{2} y_{11}^{2} y_{96}+y_{6}^{2} y_{7}^{2} y_{112} .
\end{aligned}
$$

(ii) The vertical squaring operations in $E_{2}$ are given by

$$
\begin{aligned}
& S q^{2} y_{4}=y_{6}, S q^{1} y_{6}=y_{7}, S q^{4} y_{6}=y_{10}, S q^{1} y_{10}=y_{11}, \\
& S q^{8} y_{10}=y_{18}, S q^{1} y_{18}=y_{19}, S q^{1} y_{34}=y_{35}, S q^{1} y_{66}=y_{67}, \\
& S q^{32} y_{64}=y_{96}, S q^{16} y_{96}=y_{112} .
\end{aligned}
$$

Proof. The calculation of $\operatorname{Cotor}_{A}\left(Z_{2}, Z_{2}\right)$ is the same as that given by [7], although the relations (3.10) are dropped there. Recall the outline of their calculation. Let $L=\left\{x_{3}, x_{5}, x_{3}{ }^{2}, x_{9}, x_{5}{ }^{2}, x_{17}, x_{9}{ }^{2}, x_{15}, x_{23}, x_{27}\right\}$ and denote the corresponding elements by $s L=\left\{a_{4}, a_{6}, a_{7}, a_{10}, a_{11}, a_{18}, a_{19}\right.$, $\left.b_{16}, b_{24}, b_{28}\right\}$. Then by (3.4) and (3.5) we have

$$
\begin{aligned}
& S q^{2} a_{4}=a_{6}, S q^{1} a_{6}=a_{7}, S q^{4} a_{6}=a_{10}, S q^{1} a_{10}=a_{11}, \\
& S q^{8} a_{10}=a_{18}, S q^{1} a_{18}=a_{19}, S q^{8} b_{16}=b_{24}, S q^{4} b_{24}=b_{28} .
\end{aligned}
$$

Form a differential algebra $(\bar{X}, \bar{d})$ as in $\S 2$. Explicitly

$$
\bar{X} \cong Z_{2}\left\{a_{i}, b_{j}\right\} / I, i=4,6,7,10,11,18,19, j=16,24,28,
$$

where $I$ is the ideal generated by all possible $\left[a_{m}, a_{n}\right]$ and $\left[b_{p}, b_{q}\right]$ and by $\left[a_{i}, b_{j}\right]$ except $(i, j)=(6,16),(10,16),(6,24),(10,28)$ and $\left[a_{6}, b_{16}\right]$ $+a_{11}^{2},\left[a_{10}, b_{16}\right]+a_{19} a_{7},\left[a_{6}, b_{24}\right]+a_{11} a_{19},\left[a_{10}, b_{28}\right]+a_{19}^{2}$. Then the projection $p: \bar{C}(A) \rightarrow \bar{X}$ induces an isomorphism on cohomology, i.e., $\operatorname{Cotor}_{A}\left(Z_{2}, Z_{2}\right)$ $\cong H(\bar{X}, \bar{d})$. Each $y_{i}$ is represented in $\bar{X}$ as follows.

$$
\begin{aligned}
& y_{i}: a_{i}(i=4,6,7,10,11,18,19) \\
& y_{34}: a_{18} b_{16}+a_{10} b_{24}+a_{6} b_{28}, y_{35}: a_{19} b_{16}+a_{11} b_{24}+a_{7} b_{28}, \\
& y_{66}: a_{10} b_{28}^{2}+a_{18} b_{24}^{2}+a_{19}^{2} b_{28}, y_{67}: a_{11} b_{28}^{2}+a_{19} b_{24}^{2}, \\
& y_{4 j}: b_{j}{ }^{4}(j=16,24,28) .
\end{aligned}
$$

Remark that the representative of $y_{66}$ in [7] is incorrect. Now the squaring operations on $y_{i}$ follow immediate from Corollary 2.6 and (3.11). 
Proposition 3. 3. $S q_{D}^{1} y_{34}=y_{67}$ in Cotor $_{A}\left(Z_{2}, Z_{2}\right)$, and hence $S q^{33} y_{34}$ $=y_{67}$ in $E_{2}$.

Proof. Let $C$ be a representative of $y_{34}$ in the cobar construction $\bar{C}(A)$. The explicit form of $C$ is given by

$$
\begin{aligned}
C= & {\left[x_{17} \mid x_{15}\right]+\left[x_{9} \mid x_{23}\right]+\left[x_{5} \mid x_{27}\right]+\left[x_{5} x_{17} \mid x_{5}{ }^{2}\right]+\left[x_{9} x_{17} \mid x_{3}{ }^{2}\right] } \\
& +\left[x_{5} x_{9} \mid x_{9}{ }^{2}\right] .
\end{aligned}
$$

Then $S q_{D}^{1} y_{34}$ is represented by $C \underset{1}{\cup} C$. By using the explicit formula for the cup-1-product, we have

$$
C \underset{1}{\cup} C=\left[x_{9}{ }^{2}\left|x_{23}\right| x_{23}\right]+\left[x_{5}{ }^{2}\left|x_{27}\right| x_{27}\right]+r,
$$

where $r \in \operatorname{Ker}(p: \bar{C}(A) \rightarrow \bar{X})$. Hence

$$
p(C \underset{1}{\cup} C)=a_{11} b_{28}^{2}+a_{19} b_{24}^{2} \text {. }
$$

Therefore $C \cup C$ represents $y_{67}$, and we have $S q^{1}{ }_{D} y_{34}=y_{67}$ in $\operatorname{Cotor}_{A}\left(Z_{2}, Z_{2}\right)$. The latter relation $S q^{33} y_{34}=y_{67}$ in $E_{2}$ follows from Proposition 2. 2.

q.e.d.

For later use we note

Lemma 3. 4. $S q^{1} y_{64}=S q^{2} y_{64}=S q^{4} y_{64}=0$ and $S q^{8} y_{64}=y_{18}^{4}$ in $E_{2}$

Proof. Since $S q^{1} b_{16}=S q^{4} b_{16}=0$ for dimensional reasons and $S q^{2} b_{16}$ $=a_{18}$ in $\bar{X}$ by (3.6), and since $y_{64}$ is represented by $b_{16}^{4}$, the lemma follows from Corollary 2. 6 .

q.e.d.

\section{§4. Collapsing of the Spectral Sequence for $X_{6}$}

Let $X_{6} \in\left\{E_{6} ; 2\right\}$ and put $A=H^{*}\left(X_{6} ; Z_{2}\right)$. Consider the EilenbergMoore spectral sequence for $X_{6}$ :

$$
E_{2} \cong \operatorname{Cotor}_{A}\left(Z_{2}, Z_{2}\right) \Rightarrow H^{*}\left(B X_{6} ; Z_{2}\right),
$$

where the $E_{2}$-term is given by Proposition 3.1. 
Theorem 4. 1. The Eilenberg-Moore spectral sequence (4.1) for $X_{6}$ collapses.

This will follow from the following lemmas.

Lemma 4. 2. The element $y_{1}$ survives, and hence so do $y_{6}, y_{7}$, $y_{10}, y_{18}$.

Proof. For dimensional reasons $y_{4}$ survives and so do the other elements by Propositions 1.2 and 3.1.

q.e.d.

We need the following facts.
i) $y_{18}^{2} \neq 0$ in $H^{*}\left(B X_{6} ; Z_{2}\right)$,
ii) $y_{7}^{5} \neq 0$ in $E_{3}$,
iii) $y_{4}^{2} y_{6}^{2} y_{7}^{2} \neq 0, y_{4} y_{6}^{2} y_{7}^{3} \neq 0$ in $E_{4}$,
iv) $y_{4}^{5} y_{7}^{2} \neq 0$ in $E_{5}$.

Proof is clear for dimensional reasons.

Lemma 4. 3. The element $y_{34}$ survives.

Proof. Denote $F^{p}=F^{p} H^{*}\left(B X_{6} ; Z_{2}\right)$. First note that $S q^{18} y_{18}=y_{18}^{2} \neq 0$ in $H^{*}\left(B X_{6} ; Z_{2}\right)$ by (4.2). Remark that $y_{18}^{2} \in F^{2}$. By Adem relation

$$
y_{18}^{2}=S q^{18} y_{18}=S q^{2} S q^{16} y_{18}+S q^{17} S q^{1} y_{18} .
$$

For dimensional reasons $S q^{1} y_{18} \in F^{3}$, and hence $S q^{17} S q^{1} y_{18} \in F^{3}$ by Proposition 1. 1. Now assume that $\gamma_{34}$ does not survive, then $S q^{16} y_{18} \in F^{3}$, and hence $S q^{2} S q^{16} y_{18} \in F^{3}$. This is a contradiction to $y_{18}^{2} \in F^{2}$. Thus $y_{34}$ survives and furthermore we must have

$$
S q^{16} y_{18} \equiv y_{34} \bmod F^{3} \text {. }
$$

This completes the proof.

q.e.d.

In the above proof we have shown 
Proposition 4. 4. $S q^{16} y_{18} \equiv y_{34} \bmod$ decomposables in $H^{*}\left(B X_{6} ; Z_{2}\right)$.

Lemma 4. 5. $S q^{1} y_{32}=S q^{2} y_{32}=0$ in $E_{2}$.

Proof. Recall that $y_{32}$ is represented by $b_{16}^{2}$ in $\bar{X}$ (See the proof of Proposition 3.1) and $S q^{1} b_{16}=0$ for dimensional reasons. Hence $S q^{2} y_{32}$ is represented by

$$
S q^{2} b_{16}^{2}=S q^{1} b_{16} S q^{1} b_{16}=0 \quad \text { by Corollary } 2.6 .
$$

Therefore we have $S q^{2} y_{32}=0$ in $E_{2}$. It is easier to see $S q^{1} y_{32}=0$.

q.e.d.

Lemma 4. 6. The element $y_{32}$ survives, and so does $y_{48}$.

Proof. We first show that $y_{32} \in E_{2}^{2,30}$ is a permanent cocycle. Consider $d_{r}: E_{r}^{2,30} \rightarrow E_{r}^{2+r, 31-r}(r \geq 2)$. For dimensional reasons the possible elements to be killed by $y_{32}$ are

$$
\begin{aligned}
& E_{2}^{4,29} \ni y_{4}^{2} y_{7} y_{18}=0, y_{6} y_{7} y_{10}^{2}=0, \\
& E_{3}^{5,28} \ni y_{4} y_{6}{ }^{2} y_{7} y_{10}=0, y_{6}{ }^{2} y_{7}^{3}, \\
& E_{4}^{6,27} \ni y_{4}^{4} y_{7} y_{10}=0, y_{4}^{2} y_{6}^{3} y_{7}, y_{4}^{3} y_{7}^{3}, \\
& E_{5}^{7,26} \ni y_{4}^{5} y_{6} y_{7} .
\end{aligned}
$$

Put $d_{3}\left(y_{32}\right)=a y_{6}{ }^{2} y_{7}{ }^{3}$ with $a \in Z_{2}$. Applying $S q^{2}$, we have

$$
0=d_{3}\left(S q^{2} y_{32}\right)=S q^{2} d_{3}\left(y_{32}\right)=a y_{7}{ }^{5}
$$

by Propositions 1.2,3.1 and Lemma 4.5. Then since $y_{7}^{5} \neq 0$ by (4.2) we have $a=0$. Next put $d_{4}\left(y_{32}\right)=a y_{4}{ }^{2} y_{6}{ }^{3} y_{7}+b y_{4}{ }^{3} y_{7}{ }^{3}$ with $a, b \in Z_{2}$. Applying $S q^{1}$, we have $0=d_{4}\left(S q^{1} y_{32}\right)=S q^{1} d_{4}\left(y_{32}\right)=a y_{4}{ }^{2} y_{6}{ }^{2} y_{7}{ }^{2}$. Since $y_{4}{ }^{2} y_{6}{ }^{2} y_{7}{ }^{2} \neq 0$ by (4.2), we have $a=0$. Then applying $S q^{2}$ to $d_{4}\left(y_{32}\right)=b y_{4}{ }^{3} y_{7}{ }^{2}$, we have $0=d_{4}\left(S q^{2} y_{32}\right)=S q^{2} d_{4}\left(y_{32}\right)=b y_{4} y_{6}{ }^{2} y_{7}{ }^{3}$. Since $y_{4} y_{6}{ }^{2} y_{7}{ }^{3} \neq 0$ by (4.2), we have $b=0$. Thus $d_{4}\left(y_{32}\right)=0$. Finally put $d_{5}\left(y_{32}\right)=a y_{4}{ }^{5} y_{6} y_{7}$ with $a \in Z_{2}$. Applying $S q^{1}$,

$$
0=d_{5}\left(S q^{1}\left(y_{32}\right)\right)=S q^{1} d_{5} y_{32}=a y_{4}^{5} y_{7}^{2} .
$$

Since $y_{4}{ }^{5} y_{7}{ }^{2} \neq 0$ by (4.2), we have $a=0$ and $d_{5}\left(y_{32}\right)=0$. Thus we have 
shown that $y_{32}$ is a permanent cocycle. Since $y_{32}$ is not killed for dimensional reasons, we conclude that $y_{32}$ survives, and hence $y_{48}=S q^{16} y_{32}$ survives by Proposition 1.2.

q.e.d.

Now Theorem 4. 1 follows from Lemmas 4.2, 4.3 and 4.6.

Theorems $A$ and $B$ follow immediately from Propositions 3.1 and 4. 4 and Theorem 4. 1 .

\section{§. Collapsing of the Spectral Sequence for $\mathbb{X}_{7}$}

Let $X_{7} \in\left\{E_{7}, 2\right\}$ and put $A=H^{*}\left(X_{7} ; Z_{2}\right)$. Consider the EilenbergMoore spectral sequence for $Y_{7}$ :

$$
E_{2} \cong \operatorname{Cotor}_{A}\left(Z_{2}, Z_{2}\right) \Rightarrow H^{*}\left(B X_{7} ; Z_{2}\right),
$$

where the $E_{2}$-term is given by Proposition 3. 2 .

Theorem 5. 1. The Eilenberg-Moore spectral sequence for $X_{7}$ collapses.

This will follow from the following lemmas.

Lemma 5. 2. The element $y_{4}$ survives, and so do $y_{6}, y_{7}, y_{10}, y_{11}$, $y_{18}, y_{19}$.

Proof. The element $y_{4}$ survives for dimensional reasons, and so do the other elements by Propositions 1.2 and 3.2.

q.e.d.

Lemma 5. 3. The element $y_{34}$ survives and so does $y_{35}$.

Proof is quite similar to that of Lemma 4.3, though the existence of the element of degree 19 may make a proof a little bit complicated.

As an analogous result to Proposition 4.4 we can show

Proposition 5. 4. $S q^{16} y_{18} \equiv y_{34} \bmod$ decomposables in $H^{*}\left(B X_{7} ; Z_{2}\right)$. 
Lemma 5. 5. The element $y_{66}$ survives and so does $y_{67}$.

Proof. Denote $F^{p}=F^{p} H^{*}\left(B X_{7} ; Z_{2}\right)$. By Proposition 3.3 the relation $S q^{33} y_{34}=y_{67}$ holds in $E_{2}$. Hence the element $y_{67}$ survives to $E_{\infty}$ by Proposition 1.2, and we obtain

$$
y_{67}=S q^{33} y_{34} \equiv S q^{1} S q^{32} y_{34} \bmod F^{4}
$$

in $H^{*}\left(B X_{7} ; Z_{2}\right)$. Assume that $y_{66}$ does not survive. Then $S q^{32} y_{34} \in F^{1}$ for dimensional reasons. So $y_{67} \equiv 0 \bmod F^{4}$, which is a contradiction to $y_{67} \in F^{3}$. Therefore $y_{66}$ survives and furthermore we must have

$$
S q^{32} y_{31} \equiv y_{66} \bmod F^{4} \text { q.e.d. }
$$

In the proof we have obtained

Proposition 5. 6. $S q^{32} y_{34} \equiv y_{66} \bmod$ decomposables in $H^{*}\left(B X_{7} ; Z_{2}\right)$.

Lemma 5. 7. $\quad d_{r}\left(S q^{i} y_{64}\right)=0$ for $i=1,2,4,8$ and for all $r \geq 2$.

Proof. Immediate from Lemma 3.4. q.e.d.

Lemma 5. 8. The element $y_{64}$ survives and hence so do $y_{96}$ and $y_{112}$.

(The proof will be given in $\S 6$.)

Now Theorem 5.1 follows from Lemmas 5.2, 5. 3, 5.5 and 5.8.

Theorems $C$ and $D$ follow from Propositions 3.2, 5. 4 and 5.6 and Theorem 5. 1.

\section{$\S$ 6. Proof of Lemma 5.8}

The proof of Lemma 5.8 given here is quite analogous to that of Lemma 4. 3, although it is much more complicated. To prove the lemma, it suffices to show that the element $y_{64}$ survives, since $y_{96}=S q^{32} y_{64}$ and $y_{112}=S q^{16} y_{96}$ by Proposition 3. 2. For dimensional reasons $y_{64}$ is not killed, 
and hence we need only to check that $y_{64} \in E_{2}^{4,60}$ is a permanent cocycle.

Let $S(n)$ be the set of monomials in $E_{2}{ }^{* *}$

$$
y_{4}^{a} y_{6}^{b} y_{7}^{c} y_{10}^{d} y_{11}^{e} y_{18}^{f} y_{19}^{g} y_{34}^{h} y_{35}^{i}
$$

with

(6. 2. n) $4 a+6 b+7 c+10 d+11 e+18 f+19 g+34 h+35 i=n$.

Note that the $Z_{2}$-module generated by $S(n)$ is closed under the vertical squaring operations. The set $S(n)$ is ordered lexicographically from the right, for example, $y_{4} y_{6}^{2} y_{10}^{3} y_{19}>y_{6}^{2} y_{7}^{2} y_{10} y_{19}$ in $S(65)$. Since there are relations

$$
\begin{aligned}
& y_{6} y_{11}+y_{10} y_{7}=0, y_{6} y_{19}+y_{18} y_{7}=0, y_{10} y_{19}+y_{18} y_{11}=0, \\
& y_{11}^{3}+y_{19} y_{7}^{2}=0, y_{11} y_{19}^{2}=0, y_{19}^{3}=0, y_{7} y_{34}+y_{6} y_{35}+y_{19} y_{11}^{2}=0, \\
& y_{11} y_{34}+y_{10} y_{35}+y_{7} y_{19}^{2}=0, y_{19} y_{34}+y_{18} y_{35}=0,
\end{aligned}
$$

the monomials of $S(n)$ satisfying one of the following

$$
\begin{aligned}
& \text { i) } c \geq 1, \quad d \geq 1, \quad \text { ii) } c \geq 1, f \geq 1, \text { iii) } e \geq 1, f \geq 1, \\
& \text { iv) } e \geq 3, \quad \text { v) } e \geq 1, g \geq 2, \quad \text { vi) } g \geq 3 \text {, vii) } e \geq 2, g \geq 1 \text {, } \\
& \text { viii) } c \geq 1, g \geq 2, \quad \text { ix) } g \geq 1, h \geq 1,
\end{aligned}
$$

can be reduced either to a trivial one or to a linear combination of the other monomials of higher order. A monomial is irreducible unless it satisfies one of the relations (6.3). Thus the set of the irreducible monomials of degree $n$ forms a $Z_{2}$-basis of $S(n)$.

Remark that the first (possibly) non-trivial differential is

$$
d_{r}: E_{r}^{4,80} \rightarrow E_{r}^{4+r, 81-r},
$$

since the elements $y_{i}(i=4,6,7,10,11,18,19,34,35)$ are cocycles. So the following lemmas are clear for dimensional reasons.

Lemma 6. 1. The irreducible monomials $y_{1}{ }^{a} y_{6}{ }^{b} y_{7}{ }^{c} y_{10}^{d} y_{11}^{e} y_{18}^{f} y_{19}^{g} y_{34}^{h} y_{35}^{i}$ are non-trivial in $E_{r}^{p, q}$ for the following cases:

(1) $p+q \leq 68$ and $p+q \neq 65$, when $a>0$,

(2) $p+q=69$ and 73, when $a=0$. 
Lemma 6. 2. The non-negative integer solutions of the equation (6. 2.65) and

$$
a+b+c+d+e+f+g+2 h+2 i=4+r
$$

except the cases (6.4) gives a basis $\left\{m_{r, i}\right\}$ of $E_{r}{ }^{4+r, 61-r}$.

Using this basis, each element of $E_{r}{ }^{4+r_{,} 61-r}$ is expressed as $\sum_{i} k_{i} m_{r, i}$ with $k_{i} \in Z_{2}$. Explicitly we have

i) $E_{2}^{6,59}: k_{1} y_{4}^{3} y_{18} y_{35}+k_{2} y_{4}^{2} y_{11}^{2} y_{35}+k_{3} y_{6}^{2} y_{7} y_{11} y_{35}+k_{4}, y_{4}, v_{6} y_{10}^{2} y_{35}$

$$
\begin{aligned}
& +k_{5 .} v_{4 .} v_{6} v_{10} y_{11} y_{34}+k_{8 .} y_{6 .} v_{7}^{2} v_{11} v_{34}+k_{7 .} v_{4}^{2} y_{10}^{2} y_{18} v_{14} \\
& +k_{9 .} v_{6}{ }^{3} v_{10 .} v_{19} v_{19}+k_{9 .} v_{6 .} v_{10}^{4} v_{19}
\end{aligned}
$$

ii) $E_{3}^{7,58}: k_{1 .} y_{4}^{3} y_{7} y_{11} y_{35}+k_{2} v_{4}{ }^{2} y_{6}{ }^{2} y_{10} y_{35}+k_{3} v_{4}, v_{6}{ }^{2} y_{7}{ }^{2} y_{35}+k_{4} v_{15}{ }^{5} y_{35}$

$$
\begin{aligned}
& +k_{5} y_{1}^{2} y_{6}^{2} y_{11} y_{34}+k_{6} v_{4} y_{6} y_{7}^{3} y_{34}+k_{7} y_{4}{ }^{3} y_{6} y_{10 .} v_{18 .} v_{19} \\
& +k_{8} y_{4} y_{6}^{4} y_{18} y_{19}+k_{9} y_{7}{ }^{5} y_{11} y_{19}+k_{10} y_{4} y_{6}{ }^{2} y_{10}^{3} y_{19} \\
& +k_{11} y_{4} y_{10}^{5} y_{11}
\end{aligned}
$$

iii) $\quad E_{4}^{8,57}: k_{1} y_{4}{ }^{5} y_{10} y_{35}+k_{2} y_{4}{ }^{4} y_{7}{ }^{2} y_{35}+k_{3} y_{4}{ }^{3} y_{6}{ }^{3} y_{35}+k_{4} y_{4}{ }^{5} y_{11} y_{34}$

$$
\begin{aligned}
& +k_{5} y_{4}{ }^{3} y_{6}{ }^{2} y_{7} y_{34}+k_{6} y_{4}{ }^{4} y_{6}{ }^{2} y_{18} y_{19}+k_{7} y_{4}{ }^{2} y_{6} y_{7}^{3} y_{11} y_{19} \\
& +k_{8} y_{4} y_{6}{ }^{4} y_{7} y_{11} y_{19}+k_{9} y_{4}^{4} y_{10}^{3} y_{19}+k_{10} y_{4}{ }^{2} y_{6}{ }^{3} y_{10}^{2} y_{19} \\
& +k_{11} y_{6}{ }^{6} y_{10} y_{19}+k_{12} y_{4} y_{7}^{6} y_{19}+k_{13} y_{6}{ }^{3} y_{7}{ }^{4} y_{19} \\
& +k_{14} y_{4}{ }^{2} y_{6} y_{10}^{4} y_{11}+k_{15} y_{6}{ }^{4} y_{10}^{3} y_{11}
\end{aligned}
$$

iv) $\quad E_{5}^{9,56}: k_{1} y_{4}^{6} y_{6} y_{35}+k_{2} y_{4}^{6} y_{7} y_{34}+k_{3} y_{4}{ }_{4}^{4} y_{6}^{2} y_{7} y_{11} y_{19}+k_{4} y_{4}^{5} y_{8} y_{10}^{2} y_{19}$

$$
\begin{aligned}
& +k_{5} y_{4}{ }^{3} y_{6}^{4} y_{10} y_{19}+k_{6} y_{4}^{3} y_{6} y_{7}^{4} y_{19}+k_{7} v_{4}{ }^{2} y_{6}{ }^{4} y_{7}^{2} y_{19} \\
& +k_{8} y_{4} y_{8}^{7} y_{19}+k_{9} y_{4}^{2} y_{7}^{5} y_{11}^{2}+k_{10} y_{4} y_{6}^{3} y_{7}^{3} y_{11}^{2} \\
& +k_{11} y_{6}{ }^{6} y_{7} y_{11}^{2}+k_{12} y_{4}^{3} y_{6}^{2} y_{10}^{3} y_{11}+k_{13} y_{4} y_{6}^{5} y_{10}^{2} y_{11} \\
& +k_{14} y_{8}^{2} y_{7}^{6} y_{11}
\end{aligned}
$$

v) $E_{6}^{10,55}: k_{1} y_{4}^{7} y_{7} y_{11} y_{19}+k_{2} y_{4}^{6} y_{6}^{2} y_{10} y_{19}+k_{3} y_{4}{ }^{5} y_{6}^{2} y_{7}^{2} y_{19}$

$$
\begin{aligned}
& +k_{4} y_{4}{ }^{4} y_{6}^{5} y_{19}+k_{5} y_{4}{ }_{4}^{4} y_{6} y_{7}^{3} y_{11}^{2}+k_{6} y_{4}^{3} y_{6}{ }^{4} y_{7} y_{11}^{2} \\
& +k_{7} y_{4}^{6} y_{10}^{3} y_{11}+k_{8} y_{4}^{4} y_{6}{ }^{3} y_{10}^{2} y_{11}+k_{9} y_{4}{ }^{2} y_{8}^{6} y_{10} y_{11}
\end{aligned}
$$




$$
\begin{aligned}
& +k_{10} y_{4}^{3} y_{7}^{6} y_{11}+k_{11} y_{4}^{2} y_{6}^{3} y_{7}^{4} y_{11}+k_{12} y_{4} y_{6}^{6} y_{7}^{2} y_{11} \\
& +k_{13} y_{6}^{9} y_{11}+k_{14} y_{4} y_{6}^{2} y_{7}^{7}+k_{15} y_{6}^{5} y_{7}^{5}
\end{aligned}
$$

vi) $E_{7}^{11,54}: k_{1} y_{4}{ }^{9} y_{10} y_{19}+k_{2} y_{4}{ }^{8} y_{7}{ }^{2} y_{19}+k_{3} y_{4}{ }^{7} y_{6}{ }^{3} y_{19}+k_{4} y_{4}{ }^{6} y_{6}{ }^{2} y_{7} y_{11}^{2}$

$$
\begin{aligned}
& +k_{5} y_{4}{ }^{7} y_{6} y_{10}^{2} y_{11}+k_{6} y_{4}{ }^{5} y_{6}{ }^{4} y_{10} y_{11}+k_{7} y_{4}{ }^{5} y_{6} y_{7}^{4} y_{11} \\
& +k_{8} y_{4}{ }_{4}^{4} y_{6}{ }^{4} y_{7}{ }^{2} y_{11}+k_{9} y_{4}{ }^{3} y_{6}{ }^{7} y_{11}+k_{10} y_{4}{ }^{4} y_{7}{ }^{7}+k_{11} y_{4}{ }^{3} y_{6}{ }^{3} y_{7}{ }^{5} \\
& +k_{12} y_{4}{ }^{2} y_{6}{ }^{6} y_{7}{ }^{3}+k_{13} y_{4} y_{6}{ }^{9} y_{7}
\end{aligned}
$$

vii) $E_{8}^{12,53}: k_{1} y_{4}^{10} y_{6} y_{19}+k_{2} . v_{4}^{9} y_{7} y_{11}^{2}+k_{3} y_{4}^{8} y_{6}^{2} y_{10} y_{11}$

$$
\begin{aligned}
& +k_{4} y_{4}{ }^{7} y_{6}{ }^{2} y_{7}{ }^{2} y_{11}+k_{5} y_{4}{ }^{6} y_{6}{ }^{5} y_{11}+k_{6} y_{4}{ }^{6} y_{6} y_{7}^{5} \\
& +k_{7} y_{4}^{5} y_{6}{ }^{4} y_{7}{ }^{3}+k_{8} y_{4}{ }_{4}^{4} y_{6}{ }^{7} y_{7}
\end{aligned}
$$

viii) $E_{9}{ }^{13,52}: k_{1} v_{4}{ }^{11} y_{10} y_{11}+k_{2} y_{4}{ }^{10} y_{7}{ }^{2} y_{11}+k_{3} v_{4}{ }^{9} y_{6}{ }^{3} y_{11}$

$$
+k_{4} y_{4}{ }^{8} y_{6}{ }^{2} y_{7}{ }^{3}+k_{5} v_{4}{ }^{7} y_{6}^{5} y_{7}
$$

ix) $\quad E_{10}{ }^{14,51}: k_{1} y_{4}^{12} y_{6} y_{11}+k_{2} y_{4}{ }^{11} y_{7}{ }^{3}+k_{3} y_{4}{ }^{10} y_{6}{ }^{3} y_{7}$

x) $E_{11}{ }^{15,50}: k_{1} y_{4}{ }^{13} y_{6} y_{7}$.

The above elements are the candidates to be killed off by $y_{64}$. That is,

$$
d_{r}\left(y_{64}\right)=\sum_{i} k_{i} m_{r, i} \text { with } k_{i} \in Z_{2}
$$

for $d_{r}: E_{r}^{4,60} \rightarrow E_{r}^{4+r, 61-r}(r \geq 2)$. We will show that all the coefficients $k_{i}$ are zero in the following way.

First we apply $S q^{1}$ on both sides of (6.7). Since $S q^{1} d_{r} y_{64}=d_{r} S q^{1} y_{64}$ $=0$ by Lemma 5.7 , we have

$$
\sum_{i} k_{i} S q^{1} m_{r, i}=0,
$$

where $S q^{1} m_{r, i}$ is calculated by Proposition 3.2 and by the Cartan formula. Then the linear independency of $\left\{S q^{1} m_{r, i}\right\}$ by Lemma 6.1 implies that $k_{i}=0$. By this argument we get

Lemma 6. 3. $k_{i}$ is trivial for

$$
\begin{array}{ll}
i=1,6,7,8,9 & \text { in (6.6.i), } \\
i=4,6,7,8,10,11 & \text { in (6.6. ii), }
\end{array}
$$




$$
\begin{array}{ll}
i=6,7,9,10,11,13,14,15 & \text { in (6.6. iii), } \\
i=4,5,6,8,10,12,13 & \text { in (6.6. iv), } \\
i=2,4,5,7,8,9,11,13,15 & \text { in (6.6.v), } \\
i=1,3,5,6,7,9,11,13 & \text { in (6.6. vi), } \\
i=1,3,5,6,8 & \text { in (6.6. vii), } \\
i=1,3,5 & \text { in (6.6. viii), } \\
i=1,3 & \text { in (6.6. ix), } \\
i=1 & \text { in (6.6. x). }
\end{array}
$$

Then by applying $S q^{2}$ on both sides of (6.7), we get by Lemma 5.7

$$
\sum k_{i} S q^{2} m_{r, i}=0
$$

where the summation runs over $i$ not listed in Lemma 6. 3 .

The linear independency of $\left\{S q^{2} m_{r, i}\right\}$ by Lemma 6.1 implies

Lemma 6. 4. $k_{i}$ is trivial for

$$
\begin{array}{ll}
i=3,4,5 & \text { in (6.6. i), } \\
i=3,5 & \text { in (6.6. ii), } \\
i=1,3,4,5,8,12 & \text { in (6.6. iii), } \\
i=3,11,14 & \text { in (6.6. iv), } \\
i=1,3,6,10,12,14 & \text { in (6.6. v), } \\
i=4,12 & \text { in (6.6. vi), } \\
i=2,4,7 & \text { in (6.6. vii), } \\
i=4 & \text { in (6.6. viii), } \\
i=2 & \text { in (6.6. ix). }
\end{array}
$$

Corollary 6.5. (1) $y_{4} y_{6}{ }^{2} y_{7} y_{11} y_{35}$ and $y_{4}^{3} y_{11}^{2} y_{35}$ are not trivial in $E_{r}{ }^{7,62}$.

(2) $y_{4}^{4} y_{11}^{2} y_{35}$ is not trivial in $E_{r}^{8,65}$.

(3) $y_{4}^{8} y_{6}^{2} y_{7}^{2} y_{11}$ is not trivial in $E_{r}{ }^{13,56}$.

(4) $y_{4}^{4} y_{6}^{3} y_{35}, y_{4}^{6} y_{10} y_{35}, y_{4}^{4} y_{8}^{2} y_{7} y_{34}, y_{4}^{6} y_{11} y_{34}, y_{4}^{2} y_{7}^{6} y_{19}$ are not trivial in $E_{r}^{9,80}$. 
Proof. (1) and (2): The elements $y_{6}^{2} y_{7} y_{11} y_{35}$ and $y_{4}^{2} y_{11}^{2} y_{35}$ are not $d_{r}$-images of $y_{64}$, since $k_{2}=k_{3}=0$ in (6.6.i). So $y_{4} y_{6}^{2} y_{7} y_{11} y_{35}$ and $y_{4}^{a} y_{11}^{2} y_{35}$ for $a=3,4$ are not trivial, since $d_{r}=0$ in these degrees.

(3) follows from that $k_{4}=0$ in (6.6. vii).

(4) follows from that $k_{i}=0$ for $i=1,3,4,5,12$ in (6. 6. iii).

q.e.d.

Then by applying $S q^{4}$ on the both sides of (6.7) we get the following lemma by virtue of Lemma 6. 1 and Corollary 6.5.

Lemma 6. 6. $k_{2}$ is trivial for

$$
\begin{array}{ll}
i=2 & \text { in }(6.6 . \mathrm{i}), \\
i-1,2,9 & \text { in }(6.6 . \mathrm{ii}), \\
i=1,2,7,9 & \text { in }(6.6 . \mathrm{iv}), \\
i=2 & \text { in (6.6. viii). }
\end{array}
$$

Corollary 6. 7. $y_{4}^{8} y_{7} y_{34}, y_{4}^{8} y_{6} y_{35}$ and $y_{4}^{4} y_{6}^{4} y_{7} y_{19}$ are not trivial in $E_{r}^{11,62}$.

Proof. This follows from that $k_{1}=k_{2}=k_{7}=0$ in (6.6. iv).

q.e.d.

Now we apply $S q^{8}$ on the both sides of (6.7) and by Lemma 6.1 and Corollaries 6.5 and 6.7 we get

Lemma 6. 8. $k_{i}$ is trivial for

$$
\begin{array}{ll}
i=2 & \text { in (6.6. iii), } \\
i=2,8,10 & \text { in (6.6. vi). }
\end{array}
$$

Thus we have shown that all $k_{i}$ are trivial. This completes the proof of Lemma 5.8 . 


\section{References}

[1] Adams, J. F., On the non existence of elements of Hopf invariant one, Ann. Math., 72 (1960), 20-104.

[2] Araki, S., Cohomology mod 2 of the compact exceptional groups $E_{6}$ and $E_{7}, J$. Math. Osaka City Univ., 12 (1961), 43-65.

[3] Borel, A., Sur l'homologie et la cohomologie des groupes de Lie compacts connexes, Amer. J. Math., 76 (1954), 273-432.

[4] Dold, A. and Lashof, R., Principal quasi-fiberings and fibre homotopy equivalence of bundles, Ill. J. Math., 3 (1959), 285-305.

[5] Eilenberg, S. and Moore, J. C., Homology and fibrations I, Comment. Math. Helv., 40 (1966), 199-236.

[6] Kono, A. and Mimura, M., Cohomology mod 2 of the classifying space of the compact connected Lie group of type $E_{6}, J$. Pure Appl. Algebra, 6 (1975), 61-81.

[7] Kono, A., Mimura, M. and Shimada, N., On the cohomology mod 2 of the classifying space of the 1-connected exceptional Lie group $E_{7}, J$. Pure Appl. Algebra, 8 (1976), 267-283.

[8] Milgram, R. J., The bar construction and abelian $H$-spaces, Ill. J. Math., 11 (1967), $242-250$.

[9] Moore, J. C., Algèbre homologique et homologie des espaces classificants, Sem. H. Cartan, $12(1959 / 60)$.

[10] Rothenberg, M. and Steenrod, N. E., The cohomology of classifying spaces, Bull. $A M S$, 71 (1965), 872-875.

[11] Shimada, N. and Iwai, A., On the cohomology of some Hopf algebras, Nagoya Math. J., 30 (1967), 103-111.

[12] Singer, W. M., Steenrod squares in spectral sequences, I, II, Trans. AMS, 175 (1973), 327-352.

[13] Thomas, E., Exceptional Lie groups and Steenrod squares, Michigan J. Math., 11 (1964), 151-156.

[14] Zachariou, A., A polynomial subalgebra of the cohomology of the Steenrod algebra, Publ. RIMS, Kyoto Univ., 9 (1973), 157-164. 